\title{
Análise das principais consequências do abuso sexual infanto-juvenil
}

\author{
Analysis of the main consequences of child and youth sexual abuse
}

Análisis de las principales consecuencias del abuso sexual infantil y juvenil

Amanda Apolori Tissiani ${ }^{1 *}$, Nathália de Oliveira Azevedo, Gilberto José Montaño Góes de Mendonça ${ }^{1}$, André Luís Belmiro Moreira Ramos ${ }^{1}$, Márcya Cândida Casimiro de Oliveira ${ }^{1}$, lanara Fabiana Ramalho Dias Alves ${ }^{1}$, Domennica Gomes Pecorelli', Pedro Evangelista Borges Dantas ${ }^{1}$, Layza de Souza Chaves Deininger ${ }^{1}$.

\section{RESUMO}

Objetivo: Analisar a literatura científica no que diz respeito as principais consequências do abuso sexual infantojuvenil. Métodos: Trata-se de uma revisão integrativa realizada em maio de 2021, nas plataformas MEDLINE, SciELO e LILACS, utilizando descritores e critérios de elegibilidade e exclusão para seleção do corpo amostral. Resultados: A amostra é formada por 20 artigos que versam sobre a violência sexual infanto-juvenil. Os temas abordados de forma predominante na literatura foram as repercussões causadas na vida adulta por esse tipo de violência, em $55 \%$ dos artigos, e os sinais objetivos e subjetivos que as vítimas demonstram, com $40 \%$ dos artigos. Pouco se discutiu acerca do perfil da vítima de abuso sexual infantil e sobre estratégias preventivas e terapêuticas direcionadas ao combate desse agravo, com, respectivamente, 35\% e 15\% dos artigos. Considerações finais: A vasta informação acerca das repercussões ocasionadas na vida adulta de vítimas de violência sexual infantil e sobre os indicadores objetivos e subjetivos que esses jovens podem demonstrar é essencial para criação e/ou aprimoramento de instrumentos de assistência às vítimas. Não foram identificadas atualizações sobre o perfil da vítima de abuso sexual infantil, o que indica escassez de estudos sobre esse tema.

Palavras-chave: Abuso sexual infanto-juvenil, Assistência à saúde, Profissional de saúde.

\begin{abstract}
Objective: Analyze the scientific literature regarding the main consequences of child and youth sexual abuse. Methods: This is an integrative review carried out in May 2021, on MEDLINE, SciELO and LILACS platforms, using descriptors and eligibility and exclusion criteria for selection of the sample body. Results: The sample consists of 20 articles that deal with sexual violence against children and adolescents. The themes predominantly addressed in the literature were the repercussions caused in adult life by this type of violence, in $55 \%$ of the articles, and the objective and subjective signs that victims show, in $40 \%$ of the articles. Little was discussed about the profile of the victim of child sexual abuse and about preventive and therapeutic strategies aimed at combating
\end{abstract}

${ }^{1}$ Faculdade de Ciências Médicas da Paraíba (FCMPB), Cabedelo - PB.

*E-mail: amanda.apolori@gmail.com

SUBMETIDO EM: 6/2021

ACEITO EM: 6/2021

PUBLICADO EM: 7/2021

REAS | Vol.13(7) | DOI: https://doi.org/10.25248/REAS.e8194.2021

Página 1 de 10 
this problem, with, respectively, $35 \%$ and $15 \%$ of the articles. Final considerations: The vast information about the repercussions caused in the adult life of victims of child sexual violence and about the objective and subjective indicators that these young people can demonstrate is essential for the creation and/or improvement of instruments to assist victims. No updates were identified on the profile of the victim of child sexual abuse, which indicates a scarcity of studies on this topic.

Keywords: Child and youth sexual abuse, Health care, Health care professional.

\section{RESUMEN}

Objetivo: Analizar la literatura científica sobre las principales consecuencias del abuso sexual infantil. Métodos: Se trata de una revisión integradora realizada en mayo de 2021, en las plataformas MEDLINE, SciELO y LILACS, utilizando descriptores y criterios de elegibilidad y exclusión para la selección del cuerpo muestral. Resultados: La muestra está conformada por 20 artículos que abordan la violencia sexual contra niños, niñas y adolescentes. Los temas predominantemente abordados en la literatura fueron las repercusiones provocadas en la vida adulta por este tipo de violencia, en el $55 \%$ de los artículos, y los signos objetivos y subjetivos que muestran las víctimas, en el $40 \%$ de los artículos. Poco se habló sobre el perfil de la víctima de abuso sexual infantil y sobre las estrategias preventivas y terapéuticas encaminadas a combatir este problema, con, respectivamente, el $35 \%$ y el $15 \%$ de los artículos. Consideraciones finales: La vasta información sobre las repercusiones causadas en la vida adulta de las víctimas de violencia sexual infantil y sobre los indicadores objetivos y subjetivos que pueden demostrar estos jóvenes es fundamental para la creación y / o perfeccionamiento de instrumentos de atención a las víctimas. No se identificaron actualizaciones sobre el perfil de víctimas de abuso sexual infantil, lo que indica una escasez de estudios sobre este tema.

Palabras clave: Abuso sexual infantil y juvenil, Cuidado de la salud, Profesional de la salud.

\section{INTRODUÇÃO}

A violência sexual infanto-juvenil deve ser entendida como qualquer conduta e interação erótica, homo ou heterossexual, a que estes se sujeitam, por ameaça, violência ou indução de vontade, para satisfazer desejos sexuais do agressor ou terceiro que com ele esteja (TRABBOLD VLM, et al., 2016). Desse modo, configura-se como uma realidade que pode acometer crianças e adolescentes independente da classe social, etnia ou nível educacional que possuam, impondo violação sistemática aos direitos humanos dessa população (ALVES MA, et al. 2016).

Um dos elementos para a caracterização dessa violência é o diferente estágio de desenvolvimento psicossexual entre as vítimas e o agressor, que se encontra em uma posição de poder e autoridade, razão pela qual pode ser cometido por menos de 18 anos, desde que evidente a diferença de maturidade sexual, colocando a vítima em posição de dominação em relação àquele (FRIDMAN N, et al., 2017).

Estima-se que uma a cada oito jovens no mundo já foram vítimas de algum tipo de violência; desse modo, a violência sexual em crianças e adolescentes já é reconhecida como um problema de saúde pública mundial pela Organização Mundial de Saúde (OMS), sobretudo nos países de baixa condição socioeconômica, onde esses valores aumentam sensivelmente (CHEHAB MAD, et al., 2017).

No Brasil, é o segundo tipo de violência mais comum entre crianças e adolescentes, representando um total de $35 \%$ dos casos. Fica atrás apenas do abandono e negligência, que responde a $36 \%$ do total de notificações 
registradas no Sistema de Vigilância de Violência e Acidentes (VIVA), cujas informações são colhidas da ficha individual registradas no Sistema de Informação de Agravos de Notificação (SINAN) (CHILDHOOD BRASIL, 2012).

Entretanto, os números oficiais estão longe de representar a realidade. Estima-se que menos de 10\% das violações aos direitos sexuais das crianças e adolescentes sejam percebidos pelo sistema de saúde ou polícia judiciária, em razão do que se convencionou chamar "pacto de silêncio". Esse pacto deve ser entendido como a omissão das vítimas e principalmente de seus responsáveis, que, por razões diversas, preferem silenciar-se frente às violências as quais possuem conhecimento (CHEHAB MAD, et al., 2017).

Desse modo, sendo a Atenção Básica à Saúde (ABS) a porta de entrada preferencial dos usuários de saúde, é importante que os profissionais desse nível de atenção à saúde estejam atentos aos sinais e sintomas que as crianças e adolescentes podem apresentar. Sobretudo, no âmbito da saúde da criança, estes profissionais de saúde devem se atentar aos indicadores objetivos e subjetivos de violência sexual, a fim de atuar de forma interdisciplinar frente às sequelas do abuso sexual, e tratar todas as formas de agravos que tenham acometido a criança ou adolescente, sem prejuízo de ações de promoção e prevenção à saúde (TRABBOLD VLM, et al., 2016; FRIDMAN N, et al., 2017).

Nesse sentido, com a pretensão de contribuir para o aperfeiçoamento das condutas e políticas de saúde, esse estudo tem como objetivo analisar a literatura científica no que diz respeito às principais consequências do abuso sexual infanto-juvenil.

\section{MÉTODOS}

O presente estudo se trata de uma revisão integrativa a partir de uma pesquisa do tipo revisão bibliográfica da literatura, onde foi delineada a questão norteadora - quais as principais consequências do abuso sexual infantojuvenil? - procedida a coleta de dados, estabelecimento da amostra final com base nos critérios de inclusão e exclusão, análise crítica dos estudos incluídos na revisão integrativa, discussão e interpretação dos resultados e apresentação da conclusão.

Nesse âmbito, para constituição da amostra, as buscas iniciaram no mês de maio de 2021, utilizando-se como fonte as bases de dados da Revista Literatura Latino-americana e do Caribe em Ciências da Saúde (LILACS), Scientific Electronic Library Online(SciELO) e Sistema Online de Busca e Análise de Literatura Médica (MEDLINE), sendo coletados dados dos anos compreendidos entre 2016-2021.

A busca por artigos foi promovida através do emprego de seis palavras chaves, indexadas no banco de Descritores de em Ciências as Saúde (DCS): "abuso sexual na infância", "assistência à saúde" e "profissional de saúde", "maus-tratos sexuais infantis", "violação sexual de menor" e "trabalhador da saúde" a partir da interpolação dos operadores boleanos "AND" e "OR", formando três combinações: 1 - "abuso sexual na infância AND assistência à saúde AND profissional da saúde, 2 - "maus-tratos sexuais infantis AND assistência à saúde" e 3 - "violação sexual de menor AND profissional da saúde OR trabalhador da saúde".

Os artigos encontrados foram filtrados pelos critérios de inclusão, quais sejam, apenas artigos, texto completo, idioma (português, inglês e espanhol) e período (2016 a 2021). Em seguida, realizou-se a leitura do título e resumo, descartando aqueles artigos duplicados e cujos temas escapassem a problematização proposta neste trabalho.

Os estudos que se enquadraram nos critérios de inclusão e não se fizeram descartáveis após utilização dos critérios de exclusão foram lidos na íntegra. Para a ordenação dos artigos selecionados, foi construído um fluxograma com as etapas deste presente estudo (Figura 1). 
Figura 1 - Fluxograma do corpo amostral: estratégia de busca dos artigos.

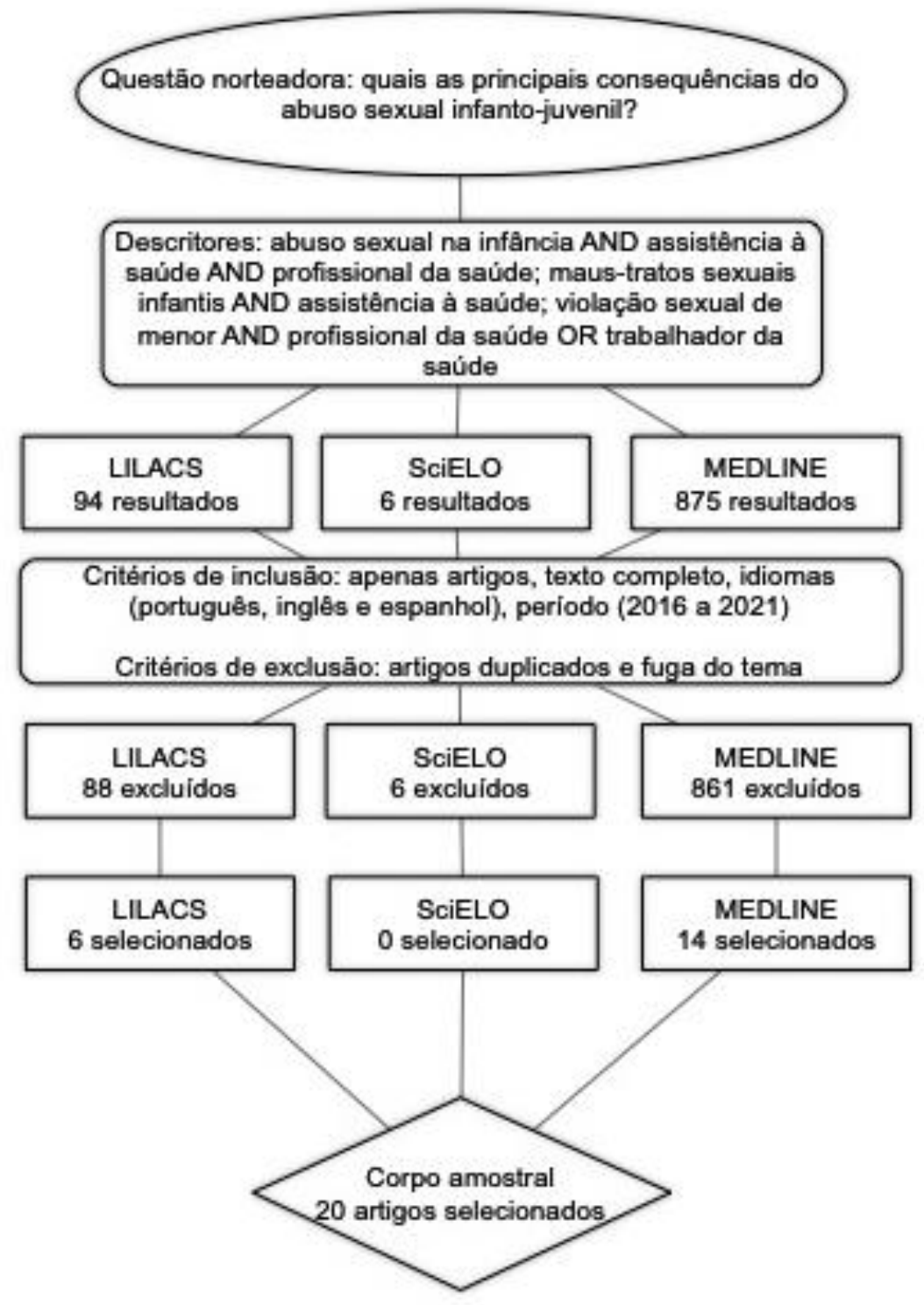

Fonte: Tissiani AA, et al., 2021.

\section{RESULTADOS}

Com a seleção dos 20 artigos, estes foram compilados no Quadro 1, a fim de facilitar a apresentação e interpretação dos resultados com os seguintes elementos: autores, título, ano de publicação, bases de dados e código em cada artigo selecionado numerado em ordem decrescente por ordem de publicação (Abuso Sexual Infanto-Juvenil - ASIJ).

Entre os artigos selecionados, 5 (25\%) foram produzidos no Brasil, 2 são de países de língua espanhola (10\%), enquanto a maioria é de países da língua inglesa (65\%). Durante o estudo de tais artigos, notou-se que os da língua inglesa trazem informações mais pertinentes sobre o assunto, abrangem mais conteúdos e envolvem mais aspectos sobre o abuso sexual em crianças e adolescentes quando comparado aos da língua portuguesa, mas que ainda são mais ricos em ensinamentos do que os da língua espanhola. 
Quadro 1 - Relação entre os autores e o título, o ano e a base de dados onde se encontra sua respectiva publicação.

\begin{tabular}{|c|c|c|c|}
\hline Autores & Título & Ano/Código & Base de dados \\
\hline GHASTINE L, et al. & Childhood Sexual Abuse - A Call to Action in Pediatric Primary Care & 2020 (ASIJ1) & MEDLINE \\
\hline JORDAN KS, et al. & $\begin{array}{l}\text { Abuso sexual pediátrico: uma abordagem interprofissional para otimizar o } \\
\text { atendimento de emergência }\end{array}$ & 2019 (ASIJ2) & MEDLINE \\
\hline KAUFMAN KL, et al. & $\begin{array}{l}\text { Recomendations for Preventing Child Sexual Abuse in Youth - Serving } \\
\text { Organizations: Implications from an Australian Royal Commission Review of the } \\
\text { Literature }\end{array}$ & 2019 (ASIJ3) & MEDLINE \\
\hline BRADY M & $\begin{array}{l}\text { UK Paramedics Confidence in Identifying Child Sexual Abuse: a Mixed Methods } \\
\text { Investigation }\end{array}$ & 2018 (ASIJ4) & MEDLINE \\
\hline WALD G, et al. & $\begin{array}{l}\text { Modelo comunitário para la promoción, prevención, asistencia y proteccion ante } \\
\text { situaciones de abuso sexual contra ninãs, niños y adolescentes }\end{array}$ & 2018 (ASIJ5) & LILACS \\
\hline FLORIAN PM & $\begin{array}{c}\text { The Unwelcome Guest: Working with Childhood Sexual Abuse Survivors in } \\
\text { Reproductive Health Care }\end{array}$ & 2018 (ASIJ6) & MEDLINE \\
\hline MASILO DT & $\begin{array}{c}\text { Prevention of Child Sexual Abuse Within the Family System: Guidelines for an } \\
\text { Educational Social Group Work Program }\end{array}$ & 2018 (ASIJ7) & MEDLINE \\
\hline FORNARI LF, et al. & $\begin{array}{l}\text { Gender and generation perspectives in the narratives of sexually abused women in } \\
\text { childhood }\end{array}$ & 2018 (ASIJ8) & MEDLINE \\
\hline OPYDO-SZYMACZEK J & $\begin{array}{l}\text { Child sexual abuse as an etiological factor of overweight and eating disorders - } \\
\text { considerations for primary health care providers }\end{array}$ & 2018 (ASIJ9) & MEDLINE \\
\hline CHEHAB MAD, et al. & $\begin{array}{c}\text { Características do abuso sexual em Santo André, São Paulo, Brasil: das vítimas ao } \\
\text { agressor, do diagnóstico ao tratamento }\end{array}$ & 2017 (ASIJ10) & LILACS \\
\hline SAMPASA-KANYINGA H, et al. & Child abuse and work stress in adulthood: evidence from a population-based study & 2017 (ASIJ11) & MEDLINE \\
\hline ZIJLSTRA E, et al. & Vulnerability and revictimization: Victim characteristics in a Dutch assault center & 2017 (ASIJ12) & MEDLINE \\
\hline WEKERLE C, BLACK T & $\begin{array}{l}\text { Gendered violence: Advancing evidence-informed research, practice and policy in } \\
\text { addressing sex, gender, and child sexual abuse }\end{array}$ & 2017 (ASIJ13) & MEDLINE \\
\hline FRIDMAN N, et al. & Abuso sexual infantil: una modalidad de abordaje interdisciplinario & 2017 (ASIJ14) & LILACS \\
\hline FONTES LFC, et al. & Violência sexual na adolescência, perfil da vítima e impactos sobre a saúde mental & 2017 (ASIJ15) & LILACS \\
\hline AL-JILAIHAWI S, et al. & The value of paediatric assessment in historic child sexual abuse & 2017 (ASIJ16) & MEDLINE \\
\hline MORRIS MC, et al. & $\begin{array}{l}\text { Community-level moderators of a school-based childhood sexual assault prevention } \\
\text { program }\end{array}$ & 2016 (ASIJ17) & MEDLINE \\
\hline TRABBOLD VLM, et al. & Concepções sobre adolescentes em situação de violência sexual & 2016 (ASIJ18) & LILACS \\
\hline ALVES MA, et al. & $\begin{array}{l}\text { Importância do cirurgião-dentista no diagnóstico de abuso sexual infantil - revisão } \\
\text { de literatura }\end{array}$ & 2016 (ASIJ19) & LILACS \\
\hline HANSON RF, ADAMS CS & $\begin{array}{l}\text { Childhood sexual abuse: identification, screening, and treatment recommendations } \\
\text { in primary care settings }\end{array}$ & 2016 (ASIJ20) & MEDLINE \\
\hline
\end{tabular}

Fonte: Tissiani AA, et al., 2021. 
Considerando os anos de publicações, constatamos que 2017 foi o ano que possui a maior quantidade de publicações, totalizando 35\% dos estudos, seguido de 2018 (30\%), 2016 (20\%), 2019 (10\%) e 2020 (5\%). Não foi encontrado nenhum artigo com o ano de 2021.

A análise temática amostra revelou que 11 (55\%) pontuam quais as repercussões causadas nas vítimas de abuso sexual. Enquanto outros oito (40\%) relatam quais são os indicadores mais frequentes para identificar uma vítima de violência sexual. Já sobre as descrições dos perfis das vítimas, somente sete (35\%) trouxeram informações relacionadas. Em número bem reduzido, apenas três (15\%) falaram sobre a estrutura adequada para assistência da vítima e das suas famílias.

\section{DISCUSSÃO}

A violência sexual contra a criança e o adolescente é um problema global de saúde pública. Com base na seleção de estudos exposta nos resultados, quatro tópicos foram elencados a fim de que sejam analisados de uma maneira mais crítica e profunda. São eles: descrição do perfil da vítima infanto-juvenil, levantamento de indicadores objetivos e subjetivos de violência sexual, a violência sexual contra a criança e o adolescente e suas repercussões e a estrutura para assistência à vítima de maus-tratos sexuais no Sistema Único de Saúde (CHEHAB MAD, et al., 2017).

$\mathrm{Na}$ descrição do perfil da vítima infanto-juvenil, um recorte de gênero revela que meninas são vítimas majoritárias deste tipo de crime, tanto na idade escolar ( 0 a 9 anos) como durante a adolescência (10 a 19 anos) e Morris MC, et al. (2016) ainda expõe em seu estud que quanto maisis jovem a criança, mais difícil dela entender e pôr em prática as formas de evitar abuso sexual. Segundo Trabbold VLM, et al. (2016), estudos internacionais chegaram ao assustador índice de que meninas são abusadas sexualmente de 1,5 a 3 vezes mais do que os meninos, havendo uma equivalência com os resultados encontrados por Alves MA (2016), quando concluiu, em seu estudo conduzido no Brasil, que a prevalência do abuso sexual em meninas é de 1 em cada 4, já em meninos é de 1 em cada 10.

Além disso, os dados expostos pela Ouvidoria Nacional de Direitos Humanos (ONDH), em 2019, revelam que em $73 \%$ dos casos a violência sexual ocorre na casa da vítima ou do suspeito, e é praticada pelo pai ou padrasto em $40 \%$ das denúncias. Dessa forma, é possível afirmar que o abuso sexual intrafamiliar é a modalidade mais frequente no território nacional. Reforçando essa ideia, Fornari LF, et al. (2018) constatou que dentre 214 vítimas dessa violência, em $48,13 \%$ dos casos foi cometida no ambiente domiciliar, em $13,55 \%$ foi perpetrada na rua e 0 restante em locais públicos como ônibus, shoppings, padarias, lojas, praias. Ainda nesse mesmo estudo, também evidenciaram que das 214 vítimas, somente $22,42 \%$ sofreram abuso por pessoas desconhecidas, ou seja, $77,58 \%$ sofreram abuso por pessoas do seu ciclo de convivência e esta realidade não conhece fronteiras.

O abuso sexual intrafamiliar muitas vezes é de difícil identificação por parte do núcleo familiar, pois o parente incestuoso pode ser visto como um "cuidador ciumento", o qual se apropria da vítima sob falso pretexto de proteção. Tal cenário favorece o processo de revitimização, ou seja, quando o menor de idade é forçado a reviver a experiência traumática (CHEHAB MAD, et. al., 2017).

Em relação à etnia e deficiências, Chehab MAD, et al. (2017) também trouxeram que, segundo o Comitê Nacional de Combate à Violência Sexual contra Crianças e Adolescente, a incidência de abuso sexual é maior em caucasianos. Além disso, nos resultados de sua pesquisa foi encontrado que entre as vítimas de abuso sexual participantes, 70,5\% eram brancas. Já Al-Jilaihawi S, et al. (2017) demonstraram em seu estudo que dentre as 249 crianças vítimas dos maus-tratos, 36 eram portadoras de deficiências como Síndrome de Down e autismo. Somado a isso, ele afirma que crianças deficientes são 3,1 vezes mais prováveis de sofrer violência sexual.

As infantes vítimas de violência sexual formam um grupo heterogêneo no que concerne ao comportamento manifestado após sofrerem a violência ou ainda estarem sendo submetidos a ela (FONTES LFC, et al., 2017). 
Em seus estudos, Hanson RF e Adam CS (2016) e Fridman N, et al. (2017), convergem quanto a apresentação de alguns comportamentos que possíveis vítimas de abuso sexual infanto-juvenil venham a apresentar. Em relação às crianças, alguns comportamentos são: ansiedade exorbitante perante separação do cuidador (geralmente a mãe), pesadelos quase todas as noites, medo repentino e exagerado de dormir no escuro, enurese noturna em crianças que anteriormente já sabiam usar o banheiro de forma adequada, tristeza ou irritabilidade, conhecimento sexual desapropriado a faixa etária e/ou uso de linguagem com conotação sexual.

Ademais, ainda sobre os estudos de Hanson RF e Adam CS (2016) e Fridman N, et al. (2017), no tocante à vítima adolescente, alguns dos comportamentos manifestados podem envolver mudanças extremas e repentinas de personalidade, isolamento, consumo de drogas ilícitas, redução do rendimento escolar, abandono escolar, transtornos dissociativos (estão associados a traumas e a estresse), transtornos alimentares, autoagressão e, em casos extremos, tentativa de suicídio. Além de manifestações comportamentais, os estudos também elencam modificações físicas que as vítimas podem apresentar, tanto na criança como no adolescente, como infecções urinárias de repetição, aparecimento de lesões genitais, dores abdominais, sangramentos repetitivos em região genitoperinal e constipação.

Para mais, Opydo-Szymaczek J, et al. (2018), através de seu estudo, acrescentam mais indicadores físicos de possível abuso sexual, como: surgimento de hematomas inexplicáveis, eritemas e/ou lacerações na área genital, anal ou nos seios de vítimas femininas, detecção de hematúria macroscópica, presença de marcas de ligadura em punhos, aparecimento de lesões defensivas em antebraços, dor anal ou vaginal podendo acompanhada ou não de prurido (OPYDO-SZYMACZEK J, et al., 2018). Além disso, de acordo Alves MA, et al. (2016), a maioria das lesões físicas está situada na região de cabeça e pescoço. Esta informação é vital, pois, cerca da metade das crianças vítimas de abuso sexual apresentam lesões em cavidade oral independente de possuírem, ou não, lesões genitais.

Dessa forma, a avaliação física da criança ou adolescente com suspeita de abuso sexual deve sempre envolver a análise da cavidade oral. Os sinais e sintomas que, quando presentes, podem levantar suspeitas de maus-tratos sexuais infantis são: a laceração de freios labiais e linguais com foco em crianças na faixa de 1 a 8 anos, presença de equimoses de sucção, diagnóstico de herpes tipo II e marcas de mordida (ALVES MA, et al., 2016). Frente ao exposto, a detecção precoce dos indicadores de violência sexual infantil permite promover a intervenção precoce. Consequentemente, o processo de recuperação física, psicossocial e comportamental das vítimas torna-se mais efetivo (AL-JILAIHAWI S, et al., 2017; BRADY M, 2018; PHYLLIS MF, 2018).

Em condições ideais, durante a infância e adolescência, o crescimento e desenvolvimento devem ocorrer em um ambiente saudável, com garantia de segurança moral, afetiva e material. Contudo, diversos autores apontam que, uma vez que o infante seja submetido à violência sexual durante o processo formativo, severas repercussões prejudicam o seu pleno desenvolvimento. Consequentemente, a vítima passa a lidar com prejuízos de ordem cognitiva, comportamental, emocional e social (MASILO DT, 2018; BRADY M, 2018; CHEHAB MAD, et. al., 2017; ALVES MA, et al., 2016; OPYDO-SZYMACZEK J, et al., 2018).

No âmbito dos malefícios de ordem psicossocial, há relatos que o primeiro sofrimento psíquico ao qual a vítima é submetida, após vivenciar o evento traumático de abuso sexual, é a decisão pela procura por assistência. As vítimas temem que seu ciclo familiar, tal qual o Sistema de Assistência à Saúde, desacreditem de seu relato. A partir desse ponto, a saúde emocional e comportamental da vítima é severamente comprometida (ZIJLSTRA E, et al., 2017). O abusado passa a assumir quadros comportamentais que, até então, não eram manifestados em seu cotidiano, como condutas agressivas súbitas, atitudes de retração e ansiedade constante (HANSON RF e ADAMS CS, 2016).

Estudos mostram que o sofrimento psíquico agudo, a longo prazo, promove repercussões psicossociais de difícil intervenção, como transtorno de estresse pós-traumático (TSPT), transtorno de ansiedade generalizada (TAG), manifestação de ideais suicidas, aumento exponencial a exposição e infecção pelo vírus HIV, elevados 
índices de abandono escolar quando comparados à indivíduos sem experiências de violência sexual e maior chance de desenvolvimento de dependência em entorpecentes como estratégia de fuga à angustia constante (CHEHAB MAD, et. al., 2017; HANSON RF e ADAMS CS, 2016; WEKERLE C e BLACK T, 2017).

Ademais, a literatura aborda o recorte de gênero como fator delimitante na avaliação da repercussão psicológica da violência sexual. Em seu trabalho, Fontes LFC, et al. (2017) apontam que homens detém menor regulação emocional quando comparados a mulheres e, por isso, apresentam mais comumente comportamentos externalizantes como forma de lidar com o trauma. Ou seja, vítima masculina de abuso sexual externa maior tendência a desenvolver problemas comportamentais. Em contrapartida, a vítima do sexo feminino, em razão da maior regulação emocional, apresenta comportamentos internalizantes, ou seja, há maior sofrimento emocional e cognitivo, podendo se manifestar como solidão e insônia.

Outrossim, ainda sob a óptica de gênero, Phyllis MF (2018) explana que mulheres com histórico de maustratos sexuais, na infância ou durante a juventude, têm maior tendência a negligenciar os cuidados de saúde preventiva, pois procuram menos por serviços de saúde. Tal cenário prejudica, em médio e longo prazo, a saúde reprodutiva desta mulher.

As principais justificativas para manutenção deste quadro incluem, primeiramente, a extrema relutância das sobreviventes de abuso sexual infanto-juvenil em se submeterem a um exame físico íntegro, principalmente quando promovido por um profissional do gênero masculino. Além disso, as vítimas relatam sentir vergonha de seus corpos, pois o associam diretamente ao sofrimento psíquico gerado pelo abuso. Há relatos de que as vítimas temem sentir dor durante exames pélvicos, por exemplo, uma vez que a algia pode engatilhar a memória sensitiva da dor física advinda da violência (PHYLLIS MF,2018).

No tocante aos prejuízos de ordem cognitiva, foi previamente citado que entre vítimas de abuso sexual há maiores índices de abando escolar. Em seu estudo, Fontes LFC, et al. (2017) expressam que indivíduos abusados irão apresentar maiores dificuldades no processo de aprendizado. Ou seja, a adaptação em ambientes escolares, universitários ou profissionais é mais complexa para a vítima de violência sexual. Consequentemente, há maiores chances que esses indivíduos dependam de programas sociais de bem-estar no futuro.

Em seu estudo, Sampasa-Kanyinga $\mathrm{H}$, et al. (2018) evidenciaram a associação significativa entre histórico de abuso sexual infantil e baixa produtividade laboral na vida adulta. O cotidiano de qualquer ocupação exige altos níveis de demanda psicológica e habilidade de lidar com diferentes graus de tensão. Todavia, adultos com história prévia de violência sexual se apresentam como pessoas fisiologicamente e psicologicamente hiper-reativas a situações de estresse. Dessa forma, esses indivíduos tendem a agir de maneira exagerada frente aos eventos rotineiros de sua rotina laboral. Por isso, quando comparados a empregados sem histórico de abuso infantojuvenil, a produtividade da vítima é menor.

Os dados apresentados anteriormente apontam que as repercussões do abuso sexual infanto-juvenil geram custos significativos para as instituições de saúde. Por isso, é vital o desenvolvimento de uma estrutura pautada em estratégias baseadas em abordagens preventivas e terapêuticas. Essas dedicam-se a promover reabilitação psicológica e a reinserção da vítima de forma produtiva na sociedade (FONTES LFC, et al., 2017). É vital que tais estratégias sejam inseridas no Sistema Único de Saúde (SUS), a fim de promover o acesso universal as essas abordagens (TRABBOLD VLM, et al., 2016).

Internacionalmente, encontram-se uma ampla variedade de modelos de programas preventivos. A princípio, são elaborados com base no público-alvo, ou seja, ou nas crianças e adolescentes ou para os cuidadores. Todavia, pelo fato de os responsáveis pela vítima serem os maiores envolvidos no abuso sexual, há uma necessidade de direcionar ações preventivas para adultos não cuidadores. Dentre esses, destacam-se profissionais da saúde da criança, como enfermeiros, médicos e odontólogos, professores e adultos públicos em geral (FONTES LFC, et al., 2017). 
A forma de abordagem também deve ser estudada. A apresentação pode ocorrer de maneira passiva, através da exposição de filmes, apresentações lúdicas e palestras, ou de uma forma ativa, por meio de participação ativa da comunidade e promoção de ensaios de comportamento protetores. A promoção de ações preventivas visa aumentar o conhecimento acerca do tema e aperfeiçoar as habilidades para enfrentar possíveis situações de abuso sexual infantil por parte da criança, do adolescente e da sociedade adulta em geral (FONTES LFC, et al., 2017).

Quanto ao programa terapêutico, as terapias cognitivo-comportamentais são o melhor método. Tal abordagem apresenta excelentes resultados na redução dos impactos negativos gerados pelo abuso sexual. $O$ transtorno de estresse pós-traumático e a sensação de ansiedade são as principais variáveis impactadas de modo benéfico. Similarmente, a terapia cognitivo-comportamental também é capaz de amenizar sintomas relacionados a desordenes psicossociais a depressão e a redução do sentimento de vergonha gerado pela violência sexual (FONTES LFC, et al., 2017).

Outrossim, através de seu estudo, Phyllis MF (2018) também sugere recomendações para o tratamento de sobreviventes do abuso sexual infantil. O objetivo principal é de promover mudança na forma como essas vítimas são tratadas no atual sistema de saúde. Para isso, primeiramente, os profissionais de saúde devem buscar adquirir domínio a respeito da identificação, diagnóstico e planejamento de conduta frente a infantes que tenham sofrido abuso.

Assim, seria adequado que esse tipo de conhecimento e sensibilidade acompanhe o profissional desde sua formação. Para isso, as escolas de medicina poderiam promover treinamento de seus alunos com médicos obstetras e enfermeiras que lidem com essa realidade. Ademais, a instituição de mecanismos que promovam o bem-estar da vítima é uma ferramenta importante para uma boa evolução. Dessa forma, a instalação de programas públicos que visem integração mente-corpo, como ioga, seria bem-quisto (PHYLLIS MF, 2018).

\section{CONSIDERAÇÕES FINAIS}

Os trabalhos analisados revelaram-se inertes no tocante ao perfil da vítima de violência sexual, uma vez que apenas reproduziram o senso comum acerca das características da criança ou do adolescente abusado. Por isso, é importante frisar a necessidade de mais pesquisas voltadas ao aprimoramento do perfil da vítima de abuso infantil. Assim, como vantagem, está presente análise tornou possível a concentração de um vasto conhecimento sobre as repercussões advindas deste tipo de violência e os indicadores apresentados pelas vítimas. Desse modo, o agrupamento deste conhecimento é de sumo importância, já que pode ser aperfeiçoado e convertido tanto em estratégias de capacitação profissional como criação ou aprimoramento de instrumentos voltados à assistência da vítima de violência sexual infantil.

\section{REFERÊNCIAS}

1. AL-JILAIHAWI S, et al. The value of paediatric assessment in historic child sexual abuse. Archives of Disease in Childhood, 2017; 102(6): 550-555.

2. ALVES MA, et al. Importância do cirurgião-dentista no diagnóstico de abuso sexual infantil: revisão de literatura. Revista Brasileira de Odontologia Legal, 2016; 3(2): 92-99.

3. BRADY M. UK Paramedics Confidence in Identifying Child Sexual Abuse: a mixed-methods investigation. Journal of Child Sexual Abuse, 2018; 27(4): 439-458.

4. CHILDHOOD BRASIL, 2012. In: Abuso sexual é o segundo tipo de violência mais comum contra crianças. Disponível em: https://www.childhood.org.br. Acesso em: 18 jun. 2021

5. CHEHAB MAD, et al. Características do abuso sexual em Santo André, São Paulo, Brasil: das vítimas ao agressor, do diagnóstico ao tratamento. Journal of Human Growth and Development, 2017; 27(2): 228-234.

6. FLORIAN PM. The Unwelcome Guest: Working with Childhood Sexual Abuse Survivors in Reproductive Health Care. Obstet Gynecol Clin North Am. 2018; 45(3): 549-562. 
7. FONTES LFC, et al. Violência sexual na adolescência, perfil da vítima e impactos sobre a saúde mental. Ciência \& Saúde Coletiva, 2017; 22(9): 2919-2928.

8. FORNARI LF, et al. Gender and generation perspectives in the narratives of sexually abused women in childhood. Revista Latino-Americana de Enfermagem, 2018; 26: e3078.

9. FRIDMAN N, et al. Abuso sexual infantil: una modalidad de abordaje interdisciplinario. Revista Del Hospital de Pediatria Garahan, 2017; 24(2): 149-154.

10. GHASTINE L, et al. Abuso Sexual Infantil: Um Chamado à Ação na Atenção Primária Pediátrica. Pediatria. 2020; 146(3): e20193327.

11. HANSON RF, ADAMS CS. Childhood sexual abuse: identification, screening, and treatment recommendations in primary care settings. Primery Care: clinics in Office Practice, 2016: 43(2): 313-326.

12. JORDAN KS, et al. Abuso sexual pediátrico: uma abordagem interprofissional para otimizar o atendimento de emergência, Revista de Enfermagem Forense, 2019; 15(1): 18-25.

13. KAUFMAN KL, et al. Recommendations for preventing child sexual abuse in youth-serving organizations: implications from an Australian Royal Commission review of the literature.Journal of Interpersonal Violence, 2019; 34(20): 4199-4224.

14. MASILO DT. Prevention of child sexual abuse whitin the family system: guidelines for an educational social group work program. Journal of Child Sexual Abuse, 2018; 27(4): 335-346.

15. MORRIS MC, et al. Community-level moderators of a school-based childhood sexual assault preventioon program. Child Abuse \& Neglect, 2017; 63:295-306.

16. OPYDO-SZYMACZEK J, et al. Child sexual abuse as an etiological factor of overweight and eating disorders: considerations for primary health care providers. Ginekologia Polska, 2018: 89(1): 48-54.

17. PHYLLIS MF. The Unwelcome Guest: Working with Childhood Sexual Abuse Survivors in Reproductive Health Care.Obstetrics and Gynecology Clinics of North America, 2018; 45(3): 549-562.

18. SAMPASA-KANYINGA H, et al. Child abuse and work stress in adulthood: evidence from a population-based study. Preventive Medicine, 2018; 108: 60-66.

19. TRABBOLD VLM, et al. Concepções sobre adolescentes em situação de violência sexual. Psicologia \& Sociedade, 2016; 28(1): 74-83.

20. WALD G, et al. Modelo comunitario para la promoción, prevención, asistencia y protección ante situaciones de abuso sexual contra niñas, niños y adolescentes. Revista de Saúde Coletiva, 2018; 28(4): e280412.

21. WEKERLE C, BLACK T. Gerend violence: advancing evidence-informed research, practice and policy in addressing sex, gender, and child sexual abuse. Child Abuse \& Neglect, 2017; 66: 166-170.

22. ZIJLSTRA E, et al. Vulnerability and revictimization: victim characteristics in a dutch assault center. Journal of Forensic and Legal Medicine, 2017; 52: 199-207. 